International Journal of Modern Physics A

(C) World Scientific Publishing Company

\title{
IMPROVED ACTION FUNCTIONALS IN NON-PERTURBATIVE QUANTUM GRAVITY
}

\author{
A. BOnANNO,${ }^{1,2}$ G. ESPOSITO $, 3,4$ C. RUBANO ${ }^{4,3}$ \\ ${ }^{1}$ Osservatorio Astrofisico, Via S. Sofia 78, 95123 Catania, Italy \\ ${ }^{2}$ INFN, Sezione di Catania, Corso Italia 57, 95129 Catania, Italy \\ ${ }^{3}$ INFN, Sezione di Napoli, Complesso Universitario di Monte S. Angelo, \\ Via Cintia, Edificio N', 80126 Napoli, Italy \\ ${ }^{4}$ Dipartimento di Scienze Fisiche, Università Federico II, Complesso Universitario \\ di Monte S. Angelo, Via Cintia, Edificio N', 80126 Napoli, Italy \\ Received 2 September 2004
}

\begin{abstract}
Models of gravity with variable $G$ and $\Lambda$ have acquired greater relevance after the recent evidence in favour of the Einstein theory being non-perturbatively renormalizable in the Weinberg sense. The present paper builds a modified Arnowitt-Deser-Misner (ADM) action functional for such models which leads to a power-law growth of the scale factor for pure gravity and for a massless $\phi^{4}$ theory in a Universe with Robertson-Walker symmetry, in agreement with the recently developed fixed-point cosmology. Interestingly, the renormalization-group flow at the fixed point is found to be compatible with a Lagrangian description of the running quantities $G$ and $\Lambda$.
\end{abstract}

Keywords: Renormalization Group; Quantum Gravity.

\section{Introduction}

The main idea of the Renormalization Group (hereafter RG) approach to field theory is to "integrate out" all fluctuations with momenta larger than some cutoff $k$, and to take them into account by means of a modified dynamics for the remaining fluctuation modes with momenta smaller than $k$. This modified dynamics is governed by a scale-dependent effective action $\Gamma_{k}$, the $k$-dependence of which is described by a functional differential equation, the exact RG equation.

Flow equations can be used for a complete quantization of fundamental theories. If the latter have the classical action $S$ one imposes the initial condition $\Gamma_{\kappa}=S$ at the ultraviolet (UV) cut-off scale $\kappa$, uses the exact RG equation to compute $\Gamma_{k}$ for all $k<\kappa$, and then sends $k \rightarrow 0$ and $\kappa \rightarrow \infty$. The defining property of a fundamental theory is that the "continuum limit" $\kappa \rightarrow \infty$ actually exists after the renormalization of finitely many parameters in the action. Interestingly, there are also perturbatively non-renormalizable theories which admit a $\kappa \rightarrow \infty$ limit. The "continuum limit" of these theories is taken at a non-Gaussian fixed point of the RG flow. It replaces the Gaussian fixed point which, at least implicitly, underlies the construction of perturbatively renormalizable theories. Recently, Lauscher and 
Reuter ${ }^{1}$ have constructed a new exact RG equation for the effective average action of Euclidean quantum gravity. They have found both a Gaussian and a non-Gaussian fixed point. A strong evidence has been therefore obtained in favour of 4-dimensional Einstein gravity being asymptotically safe. ${ }^{2}$ Here, the key question we study is how to achieve a modification of the standard ADM Lagrangian where $G$ and $\Lambda$ are dynamical variables according to a prescribed renormalized trajectory. Moreover, we are interested in the cosmological applications of such a framework. ${ }^{3}$

\section{Modified Action Functional}

We here use the ADM Hamiltonian treatment of space-time geometry. Thus, the identity

$$
\sqrt{-g}^{(4)} R=N \sqrt{h}\left(K_{i j} K^{i j}-K^{2}+{ }^{(3)} R\right)-2(K \sqrt{h})_{, 0}+2 f_{, i}^{i},
$$

where $f^{i} \equiv \sqrt{h}\left(K N^{i}-h^{i j} N_{, j}\right)$, suggests using the Leibniz rule to express

$$
\frac{1}{G}(K \sqrt{h})_{, 0}=\frac{G, 0}{G^{2}} K \sqrt{h}+\left(\frac{K \sqrt{h}}{G}\right)_{, 0}, \frac{1}{G} \frac{\partial f^{i}}{\partial x^{i}}=\frac{G, i}{G^{2}} f^{i}+\frac{\partial}{\partial x^{i}}\left(\frac{f^{i}}{G}\right),
$$

so that division by $16 \pi G$ in the integrand of the Einstein-Hilbert action yields the Lagrangian (the $c^{4}$ factor in the numerator is set to 1 with our choice of units)

$$
L=\frac{1}{16 \pi} \int\left[\frac{N \sqrt{h}}{G}\left(K_{i j} K^{i j}-K^{2}+{ }^{(3)} R-2 \Lambda\right)-2 \frac{G_{, 0}}{G^{2}} K \sqrt{h}+2 \frac{G_{, i} f^{i}}{G^{2}}\right] d^{3} x,
$$

after adding to the action functional the boundary term $I_{\Sigma}=\frac{1}{8 \pi} \int_{\Sigma} \frac{K \sqrt{h}}{G} d^{3} x$, and assuming that $\Sigma$ is a closed manifold. The Lagrangian (3), however, suffers from a serious drawback, because the resulting momentum conjugate to the three-metric reads as

$$
p^{i j} \equiv \frac{\delta L}{\delta h_{i j, 0}}=-\frac{\sqrt{h}}{16 \pi G}\left(K^{i j}-h^{i j} K\right)+\frac{\sqrt{h}}{16 \pi G} \frac{h^{i j}}{N G}\left(G_{, 0}-G_{, k} N^{k}\right) .
$$

This would yield, in turn, a Hamiltonian containing a term quadratic in $G_{, 0}$ (since $K^{i j}$ depends also on $G_{, 0} h^{i j}$ ), despite the fact that (3) is only linear in $G_{, 0}$ when expressed in terms of first and second fundamental forms. There is therefore a worrying lack of equivalence between $K^{i j}$ and $p^{i j}$. We thus decide to include a "bulk" contribution in order to cancel the effect of $G_{, 0}$ and $G_{, i}$ in Eq. (3) by writing

$$
I \equiv \frac{1}{16 \pi} \int_{M} \frac{\left({ }^{(4)} R-2 \Lambda\right)}{G(\vec{x}, t)} \sqrt{-g} d^{4} x+\frac{1}{8 \pi} \int_{M} \frac{(K \sqrt{h})_{, 0}}{G(\vec{x}, t)} d^{4} x-\frac{1}{8 \pi} \int_{M} \frac{f_{, i}^{i}}{G(\vec{x}, t)} d^{4} x,
$$

as a starting action defining a gravitational theory with variable $G$ and $\Lambda$. More precisely, upon division by $16 \pi G$ in the integrand of the Einstein-Hilbert term (first integral in Eq. (5)), the second and third integral in Eq. (5) cancel the effect of $-2(K \sqrt{h})_{, 0}$ and $2 f_{, i}^{i}$ in Eq. (1), respectively. The resulting Lagrangian density belongs to the general family depending only on fields and their first derivatives, 
which is the standard assumption in local field theory. It should also be noticed that if $G$ were constant, the second integral on the right-hand side of (5) would reduce to the York-Gibbons-Hawking boundary term $\frac{1}{8 \pi G} \int_{\Sigma} K \sqrt{h} d^{3} x$.

In other words, we are trying to generalize the standard ADM Lagrangian in order to consider $G$ as a dynamical field and investigate which dynamics is consistent with the RG approach. In this spirit we consider hereafter the following general ADM Lagrangian:

$$
L=\frac{1}{16 \pi} \int\left[\frac{N \sqrt{h}}{G}\left(K_{i j} K^{i j}-K^{2}+{ }^{(3)} R-2 \Lambda\right)\right] d^{3} x+L_{\text {int }}+L_{\text {matter }},
$$

where the first term has the same functional form as the Lagrangian of ADM general relativity (but with $G$ and $\Lambda$ promoted to dynamical variables), $L_{\text {int }}$ is an interaction term of a kinetic type which, for dimensional reasons, must be of the form (the coefficient $16 \pi$ is introduced for later convenience)

$$
L_{\mathrm{int}}=-\frac{\mu}{16 \pi} \int \frac{g^{\rho \sigma} G_{; \rho} G_{; \sigma}}{G^{3}} \sqrt{-g} d^{3} x
$$

$\mu$ being the interaction parameter. $L_{\text {matter }}$ is the "matter" Lagrangian that we shall consider as given by a self-interacting scalar field.

\section{RW Symmetry}

On using the ADM formalism, we take a scalar-field Lagrangian

$L_{m} \equiv \int \frac{N \sqrt{h}}{2}\left[N^{-2}\left(\phi_{, 0}\right)^{2}-2 \frac{N^{i}}{N^{2}} \phi_{, 0} \phi_{, i}-\left(h^{i j}-\frac{N^{i} N^{j}}{N^{2}}\right) \phi_{, i} \phi_{, j}-2 V(\phi)\right] d^{3} x,(8)$

where the potential $V(\phi)$ is, for the time being, un-determined, and $g^{00}$ is negative with our convention for the space-time metric.

We focus, hereafter, on cosmological models with RW symmetry. Strictly speaking, such a name can be criticized, since we are no longer studying general relativity, nor are we simply RG-improving the Einstein equations. Nevertheless, we will find that spatially homogeneous and isotropic cosmological models of the RW class can still be achieved. In such models with lapse function $N=1$, the full Lagrangian, including scalar field, reads as (here $\mathcal{K}=1,0,-1$ for a closed, spatially flat or open universe, respectively)

$$
L=\frac{a^{3}}{16 \pi G}\left(-6 \frac{\dot{a}^{2}}{a^{2}}+\frac{6 \mathcal{K}}{a^{2}}-2 \Lambda\right)+\frac{\mu}{16 \pi} \frac{a^{3} \dot{G}^{2}}{G^{3}}+a^{3}\left(\frac{\dot{\phi}^{2}}{2}-V(\phi)\right),
$$

where hereafter we revert to dots, for simplicity, to denote derivatives with respect to $t$. Thus, the resulting second-order evolution equations for $a, G$ and $\phi$ are

$$
\frac{\ddot{a}}{a}+\frac{1}{2} \frac{\dot{a}^{2}}{a^{2}}+\frac{\mathcal{K}}{2 a^{2}}-\frac{\Lambda}{2}-\frac{\dot{a}}{a} \frac{\dot{G}}{G}+\frac{\mu}{4} \frac{\dot{G}^{2}}{G^{2}}+4 \pi G\left(\frac{\dot{\phi}^{2}}{2}-V(\phi)\right)=0,
$$




$$
\begin{aligned}
& \mu \ddot{G}-\frac{3}{2} \mu \frac{\dot{G}^{2}}{G}+3 \mu \frac{\dot{a}}{a} \dot{G}+\frac{G}{2}\left(-6 \frac{\dot{a}^{2}}{a^{2}}+6 \frac{\mathcal{K}}{a^{2}}-2 \Lambda+2 G \frac{d \Lambda}{d G}\right)=0, \\
& \ddot{\phi}+3 \frac{\dot{a}}{a} \dot{\phi}+\frac{d V}{d \phi}=0 .
\end{aligned}
$$

Moreover, since the Lagrangian (6) is independent of time derivatives of the lapse, one has the primary constraint of vanishing conjugate momentum to $N$. The preservation in time of such a primary constraint yields, for our Lagrangian generated from the assumption of RW symmetry, the constraint equation

$$
\frac{\dot{a}^{2}}{a^{2}}+\frac{\mathcal{K}}{a^{2}}-\frac{\Lambda}{3}-\frac{\mu}{6} \frac{\dot{G}^{2}}{G^{2}}-\frac{8 \pi G}{3}\left(\frac{\dot{\phi}^{2}}{2}+V(\phi)\right)=0 .
$$

In cosmology, the postulate of homogeneity and isotropy implies that the RG scale dependence is turned into a time dependence: $G(t) \equiv G(k=k(t)), \Lambda(t) \equiv$ $\Lambda(k=k(t))$. The explicit law is $k(t)=\xi / t, \xi$ being a positive constant, because, when the age of the Universe is $t$, no fluctuation with a frequency smaller than $1 / t$ can have played any role as yet. Hence the integrating-out of modes which underlies the Wilson renormalization group should be stopped at $k \approx 1 / t$. In the neighbourhood of a fixed point $\left(g_{\star}, \lambda_{\star}\right)$ the evolution of the dimensionful $G$ and $\Lambda$ is approximately given by $G(k)=\frac{g_{\star}}{k^{2}}, \Lambda(k)=\lambda_{\star} k^{2}$. We therefore obtain the time-dependent Newton parameter and cosmological term:

$$
G(t)=g_{\star} \xi^{-2} t^{2}, \quad \Lambda(t)=\frac{\lambda_{\star} \xi^{2}}{t^{2}} .
$$

Unlike models where only the Einstein equations are RG-improved, our framework allows for a non-trivial dynamics of the scale factor even in the absence of coupling to an external field. ${ }^{4}$ To appreciate this point, consider first the case when no scalar field exists, so that Eq. (12) should not be considered. The relation (14) suggests looking for power-law solutions of the type

$$
a(t)=A t^{\alpha}, \quad G(t)=g_{\star} \xi^{-2} t^{2}, \quad \Lambda(t)=\lambda_{\star} \xi^{2} t^{-2},
$$

and separately consider the $\mathcal{K}=0$ and $\mathcal{K}= \pm 1$ case. For $\mathcal{K}=0$ we obtain that $A$ is an un-determined factor, while (from Eq. (13))

$$
\mu_{ \pm}=\frac{1}{4}\left(3 \pm \sqrt{9+12 \xi^{2} \lambda_{\star}}\right), \quad \alpha_{ \pm}=\frac{1}{6}\left(3 \pm \sqrt{9+12 \xi^{2} \lambda_{\star}}\right),
$$

which implies a power-law inflation for the "+" solution, $\alpha_{+}$being larger than 1 if $\lambda_{\star}>0$, and a possible solution of the horizon problem. Note that the first equality is a relation between coupling constants which has to be satisfied, while the second simply relates the value of $\alpha$ with the product $\xi^{2} \lambda_{\star}$. Since $\xi$ is not determined, $\alpha_{+}$ can be made arbitrarily large. Moreover, both $\Omega_{\mu}$ and $\Omega_{\Lambda}$ are constant, since

$$
\Omega_{\mu}=1-\Omega_{\Lambda}=\frac{6}{3 \pm \sqrt{9+12 \xi^{2} \lambda_{\star}}} .
$$


If $\mathcal{K}= \pm 1$ we find instead $\alpha=1$ and

$$
\mu=\frac{1}{4}\left(6+\xi^{2} \lambda_{\star}\right), \quad \frac{\mathcal{K}}{A^{2}}=\frac{\xi^{2} \lambda_{\star}}{2},
$$

where, as before, the former equation relates the values of the coupling constants, while the latter is a consistency relation. In particular we see that, if $\mathcal{K}=-1$, then $\lambda_{\star}$ must be negative. In both cases (i.e. $\mathcal{K}= \pm 1$ ), $\Omega_{\Lambda}$ and $\Omega_{\mu}$ are constant with

$$
\Omega_{\Lambda}=\frac{2 \mathcal{K}}{3 A^{2}}, \quad \Omega_{\mu}=1+\frac{\mathcal{K}}{3 A^{2}} .
$$

Next we consider the contribution of a scalar field with a self-interacting potential of the type $V(\phi)=\frac{\zeta}{4 !} \phi^{4}$, with the ansatz (15) for $a, G, \Lambda$, and $\phi=\phi_{0} t^{-\beta}$ for the scalar field. The Klein-Gordon equation of motion (12) then yields $\beta=1$ and

$\phi_{0}= \pm \sqrt{\frac{6(3 \alpha-2)}{\zeta}}$, which implies $\alpha>2 / 3$ so as to have a real scalar field. We then find, if $\mathcal{K}=0$,

$$
\begin{aligned}
& \alpha_{ \pm}=\mu \pm \sqrt{\mu^{2}-\frac{4 \mu}{3}-\frac{2 \xi^{2} \lambda_{\star}}{3}} \\
& \zeta_{ \pm}=\frac{24 g_{\star} \pi\left[3 \xi^{2} \lambda_{\star}-3 \mu^{2}+5 \mu \pm(1-\mu) \sqrt{3 \mu(3 \mu-4)-6 \xi^{2} \lambda_{\star}}\right]}{\xi^{2}\left(3 \xi^{2} \lambda_{\star}+2(3-2 \mu) \mu\right)},
\end{aligned}
$$

where (21) is a consistency relation, (20) determines the value of $\alpha$, and a reality condition for $\xi^{2} \lambda_{\star}$ is given by $\left(3 \xi^{2} \lambda_{\star}+2(3-2 \mu) \mu\right)>0$. It is not difficult to see that there are physically interesting solutions with power-law inflation if $\mu$ is large enough and positive, and $\xi$ is positive. If instead $\mathcal{K}= \pm 1$ the only solution is for $\alpha=1, \beta=1$, and we get the consistency conditions ${ }^{4}$

$$
\mu=-\frac{9 \pi \xi^{-2} g_{\star}}{\zeta}+\frac{1}{4}\left(6+\xi^{2} \lambda_{\star}\right), \quad \frac{\mathcal{K}}{A^{2}}=\frac{6 \pi \xi^{-2} g_{\star}}{\zeta}+\frac{\xi^{2} \lambda_{\star}}{2}, \quad \phi_{0}= \pm \sqrt{\frac{6}{\zeta}} .
$$

A challenging open problem is now the development of cosmological perturbation theory starting from Lagrangians as in Eq. (6). This could tell us whether formation of structure in the early universe can also be accommodated within the framework of a Wilson-type ${ }^{5}$ formulation of quantum gravity.

\section{Acknowledgments}

The authors are grateful to the INFN for financial support. The work of G. Esposito has been partially supported by PRIN 2002 "Sintesi".

\section{References}

1. O. Lauscher and M. Reuter, Phys. Rev. D65, 025013 (2002).

2. S. Weinberg, in General Relativity, an Einstein Centenary Survey, eds. S. W. Hawking and W. Israel (Cambridge University Press, Cambridge, 1979).

3. A. Bonanno and M. Reuter, Phys. Rev. D65, 043508 (2002).

4. A. Bonanno, G. Esposito and C. Rubano, gr-qc 0403115

5. K. G. Wilson and J. B. Kogut, Phys. Rep. 12, 75 (1974). 